\title{
Cardiac channelopathies - A too frequently trivialized problem?
}

\author{
Marcin Urbańczuk' ${ }^{1}$ Andrzej Jaroszyński ${ }^{1}$ \\ ${ }^{1}$ Chair and department of Family Medicine, Medical University, Lublin, Poland
}

Urbańczuk M, Jaroszyński A. Cardiac channelopathies - A too frequently trivialized problem? J Pre-Clin Clin Res. 2015; 9(1): 69-73. doi: $10.5604 / 18982395.1157580$

\begin{abstract}
Channelopathies are a genetically conditioned group of disorders related with defects in ion channels, responsible for the occurrence of dangerous abnormal heart rhythm in a structurally normal heart. These diseases are a frequent cause of arrhythmias and sudden cardiac death, especially among the young population. The following congenital channelopathies are discussed in the presented study: long QT syndrome (LQTS), Brugada syndrome, short QT syndrome (SQTS), and catecholaminergic polymorphic ventricular tachycardia (CPVT). The symptoms most often occur during physical effort or great stress, but may also occur while resting. The diagnosis is based on medical and family history taking, ECG recording, and sometimes on genetic tests. These diseases are relatively prevalent and are characterized by high mortality. Unfortunately, the treatment remains practically exclusively symptomatic. Pharmacotherapy and implantable cardioverter-defibrilator (ICD) are used to prevent sudden cardiac death. In some centres worldwide, surgical left cardiac sympathetic denervation (LCSD) is performed in patients with contraindications for other forms of therapy.
\end{abstract}

\section{Key words}

channelopathies, sudden cardiac death, long QT syndrome, short QT syndrome, Burgada syndrome

\section{INTRODUCTION}

Sudden cardiac death (SCD) is an important problem of contemporary public health. Every year worldwide, 4-5 million people die from this mechanism [1]. Precise statistics concerning this problem are not unequivocally known, because in the majority of cases a full diagnosis of SCD may be made not earlier than based on retrospective examinations. In addition, over $60 \%$ of SCD events take place without witnesses, for example, during sleep [2]. SCD is defined as an unexpected death preceded by the loss of consciousness that occurs within 1 hour from the onset of symptoms. A considerable percentage of these patients had not been previously diagnosed with any heart disease. The main cause of sudden cardiac death in people aged over $35-40$ is coronary disease. In younger patients, congenital defects occupy the primary position, including ion channels disorders called channelopathies. They lead to abnormal heart rhythm and death during their course. Channelopathies are a heterogeneous group which covers, among others, long QT syndrome (LQTS), short QT syndrome (SQTS), Burgada syndrome, and catecholaminergic polymorphic ventricular tachycardia (CPVT). Channelopathies are determined by many various mutations which are translated into difficulties in their diagnostics and treatment. Moreover, mutations in genes which are responsible for these disorders are characterized by various penetration; thus, the same mutation may be manifested in a different way in individual family members [3]. Unfortunately, not all cases of SADS caused by channelopathy are successfully fully diagnosed. It is estimated that using available methods it is possible to confirm $60-70 \%$ of the cases of LQTS, and only $20 \%$ of cases of Burgada syndrome [4]. This is especially important, because in the case of positive test results it is possible to cover

Address for correspondence: Marcin Urbańczuk, Chair and department of Family Medicine, Medical University, Staszica 11, 20-081 Lublin, Poland

E-mail: marcin_urbanczuk@wp.pl

Received: 24 February 2015; accepted: 26 May 2015 with prophylactic examinations also the patient's family members who do not show any symptoms. The effects of abnormal heart rhythm in the course of channelopathy may be dramatic, especially among the young, seemingly healthy individuals. Therefore, it is very important to keep them in mind and under consideration in the differential diagnostics of syncopal and cardiac events.

Pathogenesis. Channelopathies are a group of disorders affecting the heart muscle on a molecular level. They concern ion channels which are responsible for the release and normal dispersion of electric potential $[5,6]$. They are most frequently encountered in young individuals without structural cardiac disease. Mutations of genes encoding ion channels sub-units cause changes in triggering and the course of the functional potential of cardiac muscle cells which, in consequence, affects the body surface ECG recording. Various phenotypic penetration of mutated genes results in a very heterogeneous image of disorders found in the ECG - from those evident to very discrete, or even their lack. The latter may be manifested with the presence of an additional triggering factor - e.g. a drug, inflammatory state, electrolytic disorders or great stress [7].

The majority of known channelopathies may be suspected in the case of obtaining characteristic changes in the ECG recording. Therefore, a comprehensive analysis is very important. Ion channels defects are manifested by abnormalities in the repolarization of cardiomyocytes. They may cause a shortening or prolongation of the QT segment, changes within T wave, or various changes in the ST segment. There occurs the phenomenon of spatial dispersion of repolarization between the right and left ventricles or apical cells and the base of the heart, and the development of the phenomenon of transmural non-heterogeneity (between the endocardium and epicardium). The consequence of this phenomenon is an unequal dispersion of the depolarization wave in the heart, which constitutes a basis for life-threatening polymorphic tachycardia and ventricular fibrillation [8]. 
Long QT syndrome (LQTS). The long QT syndrome (LQTS) is defined as a congenital arrhythmogenous disorder associated with a prolongation of the QT interval on ECG recordings (Fig. 1), caused by mutation of the ion channels. The normal length of corrected QT interval (QTc) is: in males $-0.43 \mathrm{~s}$, females $-0.45 \mathrm{~s}$, and children aged $1-15-0.44 \mathrm{~s}$. The above-mentioned values should provide an incentive for seeking the cause for this state. A person affected by the disease most often does not show any symptoms, or presents various cardiac rhythm abnormalities in the form of syncopes, and sudden, often short-lasting cardiac arrest (MAS syndrome), or SCD [9].

The LQTS may also be inherited autosomatically recessive (Jarvell-Lange-Nielsen) or dominant (Romano-Warda syndrome). The latter is significantly more often observed and covers $99 \%$ of all cases of LQTS. The Jarvell-LangeNielsen syndrome is accompanied by congenital deafness. Considerably more rare types of LQTS are Andersen syndrome and Timothy syndrome in which, apart from the prolongation of QT, there also occur many non-cardiac symptoms [10]. To-date, 12 genes have been discovered the mutations of which condition the development of LQTS. The genotypes marked LQT1 - LQT3 are the most frequent. Genes, the mutations of which cause LQT1 and LQT2, encode potassium channels proteins, whereas LQT3 is the effect of mutation in the gene encoding sodium channels proteins.

The most common form of LQTS is the genotype LQT1, which is found in approximately $50-60 \%$ of patients. Cardiac rhythm abnormalities developing during physical effort are most characteristic for the type LQT1, and have been most often observed during swimming, diving, and extreme sports. Patients with this type of LQTS best respond to the therapy with beta-blockers, which is effective in about $75 \%$ of cases. In patients with type LQT2, cardiac events are most frequently observed during sleep and resting, and are specifically induced by an alarm clock, siren, or doorbell. Patients with LQT2 also respond relatively well to therapy with beta blockers, and additional supplementation of potassium is beneficial in these patients. Patients with LQT3 experience cardiac events most often during resting or sleep. In this group, therapy with beta blockers is the least effective [11]. The Schwartz scale is useful in making the diagnosis (Tab. 1).
Table 1. Diagnostic criteria of long QT syndrome according to Schwartz [12]

Electrocardiographic variables *

QTc interval acc. to Bazett's formula: > $480 \mathrm{~ms} 460-470 \mathrm{~ms} 450 \mathrm{~ms}$

Torsade de pointes** 2

T wave alternans

Notched T waves in 3 leads

Resting bradycardia (below the 2nd percentile for age)

Data from medical history

Syncope during stress (effort)**

Syncope without stress (effort)**

1

Congenital deafness

0.5

Data from family history

LQTS in the family 1

Sudden cardiac death among first-degree relatives aged below 30 0.5

*after exclusion of the effect of medicine used or diseases taking course with changes belowmentioned changes in electrocardiogram

**mutually exclusive (in accordance with symptoms only one of the possibilities is considered, or the most frequently occurring situation)

Scoring:

1 score or less - low probability of LQTS

$1.5-3$ scores - mediocre probability of LQTS

3.5 scores and more - high probability

According to the guidelines in effect HRS/EHRA/APHRS Expert Consensus 2013, LQTS is diagnosed after excluding the secondary causes of tprolongation of the QT, when:

1. a) scoring according to diagnostic criteria LQTS (19932011; Tab. 1) is $\geq 3.5$

and/or

b) diagnosed presence of one of the pathogenic mutations in one of the genes responsible for LQTS

or

c) QTc $\geq 500 \mathrm{~ms}$ (using Bazett's formula) in the subsequent 12-lead ECG

2. QTc $480-499 \mathrm{~ms}$ in the subsequent 12-lead ECG, when there occur unexplained synocopes (even when the result of genetic test is negative) [13].

The measurement of QT interval is performed from the beginning of the $\mathrm{Q}$ wave to the end of the $\mathrm{T}$ wave. The most accurate measurement is obtained in the lead with the longest QT interval at a paper speed of $25 \mathrm{~mm} / \mathrm{s}$. This is usually limb lead II or the lead with the most distinct (tallest) T wave. The
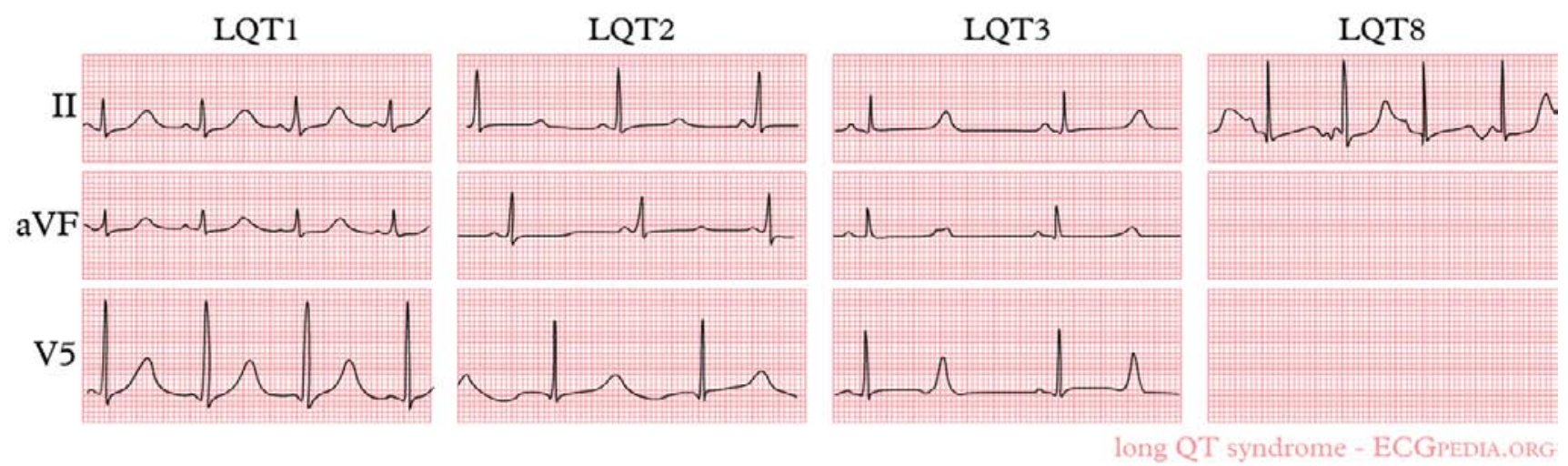

Figure 1. ECG recording typical of LQTS syndrome 
measurement should end at the point where the downward slope of the $\mathrm{T}$ wave crosses the isoelectric line. This may be difficult in practice due to the heterogeneity of T-wave morphology, and the presence of the $\mathrm{U}$ wave. When the $\mathrm{T}$ wave is overlapping on the $U$ wave, as the end of the QT interval is considered the point at which the tangent to the downward slope of the $T$ wave crosses the isoelectric line. The result should be the mean value from several evolutions of the ECG. The QT interval depends on the heart rate; therefore, the result obtained should be corrected for this rate. Specially developed formulae, the most popular of which is Bazett's formula $(\mathrm{QTc}=\mathrm{QT} / \sqrt{\mathrm{RR}})$, serve for this purpose serve for this purpose. At present, QTc is automatically calculated by the electrocardiograph. Manual measurement prevails over the automatic. The device has problems with calculation of QTc in the case of abnormal morphology of the $\mathrm{T}$ wave, presence of the $U$ wave, or simply interferences in the recording itself which often occurs in children. In addition, various producers apply different algorithms for the calculation of QTc by electrocardiogram, which is translated into the differences in results. The QT interval is not the same in all leads.

At present, the treatment of prolonged QT syndrome is based primarily on symptomatic treatment. The management is based on the avoidance of drugs acting on the prolongation of the QT interval, to which belong, among others, some of the anti-arrythmic drugs, especially those of class IA and III (sotalol, amiodarone), neuroleptics, anti-depressants, anti-biotics, and anti-histamine drugs. In the case of a patient with LQTS, it is always necessary to check whether the drug applied does not prolong the QT interval. The characteristics of the therapeutic product many be used, or the Internet databases, e.g. www.qtdrugs.org. It is also important to avoid situations which may cause arrhythmia, thus, an intensive physical effort, stress, and competitive sports. People with LQTS should avoid loud sound signals, because they may stimulate the occurrence of arrhythmia. The patient should be instructed to turn down the volume of such devices as telephone, alarm clock, and even a doorbell. It is important for these patients to take drugs decreasing the level of potassium, magnesium and calcium. They should have a regular check-up of the concentration of electrolytes, and a constant supplementation implemented as needed.

The treatment of LQTS is based on the application of betaadrenolytic drugs. Non-selective drugs are preferable, such as proplanolol or nadolol in a maximum dose tolerated by the patient. Selective drugs, such as metoprolol, reduce the occurrence of arrhythmia; however, they do not prevent sudden cardiac death (SCD). In patients with a past history of an episode of cardiac arrest, or who despite the application of beta blockers, had episodes of syncopes and ventricular tachycardia, the treatment of choice is the implantation of an implantable cardioverter defibrillator (ICD). In exceptional cases, in patients with prolonged QT and risk factors, this may be the first-line therapy. In patients who despite treatment continue to show symptoms, left cardiac sympathetic denervation (LCSD) is applied. It is performed using thoracoscopy and the least invasive methods possible [14].

Brugada syndrome. In 1992, the Brugada brothers described several cases of SCD which occurred in people without an organic heart disease. In all patients the ECG recording was similar. It covered the block of the right branch of the bundle of His, and an elevation of the ST segment (Fig. 2) [15].

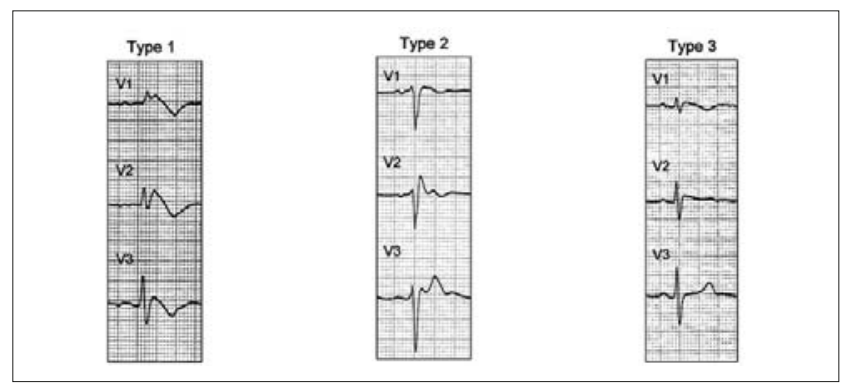

Figure 2. Examples of ECG recordings typical of Brugada syndrome (Types 1, 2, 3).

Since that time, it has been proved that this syndrome is caused by a sodium channels defect, inherited autosomally, dominant with an incomplete penetrance of the gene. It is believed that Brugada syndrome is heterogenous and may be related with approximately 300 mutations, including 12 genes. The most common mutation occurring in about $20 \%$ of cases is mutation in the SCN5A gene (Type 1 Brugada syndrome). The incidence of Brugada syndrome is estimated at $5-66$ cases per 10,000 population. This disease is relatively uncommon; however, it may be encountered in the practice of a physician of any specialty. An early diagnosis of Brugada syndrome is of fundamental importance for a patient, because treatment undertaken before it comes to cardiac arrest saves the patient's life $[16,17]$. The diagnostics of Brugada syndrome are based on the assessment of the standard ECG recording. Unfortunately, in the majority of cases, the changes are latent and occur only periodically. In the cases of unexplained loss of consciousness, tests provoking syncope may be useful with the use of sodium channel antagonists (e.g. ajmaline, propafenone), which cause the conversion of ECG to diagnostic recording. The drugs which may disguise the latent changes in ECg characteristic of Brugada syndrome also include: beta-blockers, sodium channel blockers, nitrates and psychotropic drugs. In addition, the characteristic changes in the ECG recording may be observed during intoxication with alcohol or cocaine. Electrocardiograpohic criteria of the Brugada syndrome cover:

1. elevation of the ST segment at point J by at least $2 \mathrm{~mm}$ in leads V1-V2 (V3);

2. lack of known other cause of elevation of the ST segment; block of the right branch of the bundle of His is strongly associated with this syndrome; however, its presence is not necessary to make a diagnosis [18].

During differential diagnostics, the patient's family medical history should be considered (arrhythmias, sudden deaths at a young age), previously occurring syncopes and documented events of ventricular tachycardia or ventricular fibrillation. The main cause of ST segment elevation in the leads V1-V3 are acute coronary syndromes. Table 2 presents other causes.

In a person with the diagnosis of Brugada syndrome, electrophysiological examination is usually performed in order to stratify risk. In the majority of cases, the treatment of patients with Burgada syndrome consists in a constant observation, according to the risk of implantation of cardioverter-defibrillator (ICD), in order to prevent sudden cardiac death. In pharmacological treatment a favourable effect of isoproterenol, dysopiramide and orciprenaline has been proved; however, these drugs are not routinely applied. 
Table 2. Possible causes of elevation of ST segment in leads V1-V3 [19]

TAortic dissecting aneurysm

Hypercalcaemia

Inflammation of heart muscle

Hyperkalaemia

Intoxication with cocaine

Thiamine deficiency

Overdosing heterocyclic drugs

Antidepressants

Mediastinal tumour compressing the right ventricle

Arrhythmogenic right ventricular dysplasia

Long QT syndrome

Early repolarization syndrome

Pulmonary embolism

Normal variant

Patients should also be informed concerning the possible negative effect of medicines on the course and complications of their disease (list available on website www.brugadadrugs. org). In addition, the patient's closest family should be examined. A person afflicted by Brugada syndrome should remain under constant cardiologic care.

Short QT syndrome. The short QT syndrome (SQTS) is a genetically conditioned disease of the heart characterized by an abnormally short QT interval. In 2003, it was proved that this syndrome is inherited autosomally dominant, and its pathogenesis is related with mutation of genes responsible for the normal functioning of potassium (KCNH2, KCNQ1, KCNJ2) and calcium channels (CACNA1C, CACNB2b) [20, 21]. This is a relatively rare and poorly recognized pathological entity. In the family history of a patient with short QT syndrome, information may be found concerning syncopes, arrhythmias, and sudden deaths, which occurred despite the lack of unequivocal causes. The value QTc $<300-330 \mathrm{~ms}$ was adopted as the cut-off point of short QT. However, it should be remembered that a short QT interval may simply reflect the external factors affecting ECG morphology, such as foxglove, hypercalcaemia, hyperkaliemia, acidosis, acceleration of heart rate or autonomic system disorders, and is therefore not an actual short QT syndrome.

A considerable number of SQTS cases are manifested already at a young age by sudden cardiac arrest; moreover, it is presumed that this syndrome is partly responsible for cases of sudden infant death. Giustetto et al. published the results of the largest study worldwide concerning SQTS, which covered 53 patients with SQTS, at mean age 26, observed for 64 months [22]. Nearly $90 \%$ of patients in the study experienced cardiac arrest, or it occurred in the closest family, and in $1 / 3$ this was the first clinical manifestation of the disease. In $15 \%$, syncopes were observed caused by episodes of VT or VF. Arrhythmia events occurred both at rest and during effort. In $15 \%$ of patients, AF was also registered. In order to diagnose SQTS the criteria may be used proposed by the consensus of experts HRS/EHRA/APHRS 2013 (Tab. 3).
Table 3. Valid SQTS criteria according to experts HRS/EHRA/APHRS 2013 [13]

Duration of QTc interval $<330 \mathrm{~ms}$

SQTS syndrome may be diagnosed if QTc $<360 \mathrm{~ms}$ and there coexists one of the below-mentioned characteristics:

Pathogenic gene mutation

Short QT syndrome in a family

Sudden cardiac death $<40$ year of life

Past episode of VT/VF without concomitant cardiac disease

The treatment consists in a precise monitoring and implementation of ICD in patients without contraindications. In the above-quoted study it was also found that in the patients with SQTS treated with chinidine, no episode of arrhythmia was observed. Thus, this drug may constitute a therapeutic option when the ICD cannot be implanted.

Catecholaminergic polymorphic ventricular tachycardia. Catecholaminergic polymorphic ventricular tachycardia (CPVT) is a disease related with the mutation of ryanodine receptor (RyR2) gene, responsible for intracellular calcium ions metabolism. A more rare form of CPVT, it is related with calsequestrin mutations (CASQ), protein binding calcium ions inside the sarcoplasmic reticulum. These mutations cause a constant flow of calcium ions from the sarcoplasmic reticulum to the cytoplasm, increasing the activity of sodium-calcium transporter, resulting in the development of late potentials. During adrenergic stimulation (by stress, physical effort or strong emotions) of the RyR2 receptor, the level of calcium in a cell rapidly increases, leading to the development of life-threatening ventricular tachycardia. The incidence in Europe is estimated at approximately 1/10,000. CPVT may be the cause of about $14 \%$ of SCD [23]. The symptoms usually occur in childhood. Typically, CPVT occurs in the form of bi-directional ventricular tachycardia, but may also cause supraventricular teachycardia and atrial fibrillation. Patients who experience syncopes and abnormal heart rhythm during effort, without an organic heart disease and with a burden of the disease in family history, should be subjected to an exercise test (frequently repeated arrhythmias may be induced) and examination by the Holter ECG monitoring system. The treatment of choice in patients with CPVT is the use of non-selective beta blockers. In the diagnostics and differentiation of CPVT, a pharmacological test with adrenaline may be useful. In the case of CPVT, a trial of adrenaline $(0.1 \mathrm{mg} / \mathrm{kg} / \mathrm{min}$ i.v. $)$ induced ventricular arrhythmia typical of CPVT, while in the case of LQTS, paradoxically, the prolongation of the QT interval [24]. In patients with CPVT, physical activity should be considerably limited. Implementation of ICD is also recommended in order to prevent sudden cardiac death. In some cases, cardiac sympathetic denervation and treatment with flecanide may be beneficial.

\section{CONCLUSIONS}

Channelopathies are a large heterogenous group of diseases. They are relatively rare disorders characterized by high mortality among the young, seemingly healthy population. Frequently, their first manifestations are syncopes and 
abnormal heart rhythm, and patients first seek the assistance of a family physician. Therefore, in everyday practice, it is worth remembering channelopathies, because an early diagnosis and appropriate treatment may increase the average life span of these patients.

\section{REFRENCES}

1. González-Melchor L, Villarreal-Molina T, Iturralde-Torres P, MedeirosDomingo A. Sudden cardiac death in individuals with normal hearts: an update. Arch Cardiol Mex. 2014; 84: 218-223.

2.Wiśniewski S, Kordel K, Olasiński J, Olasińska-Wiśniewska A, Wiśniewska-Śliwińska H, Marcinkowski J. Nagły zgon sercowy w materiale sekcyjnym Katedry i Zakładu Medycyny Sądowej Uniwersytetu Medycznego im. K. Marcinkowskiego w Poznaniu w latach 2001-2005. Orzecznictwo Lekarskie 2010; 7(2): 94-98 (in Polish).

3. Kaab S, Schulze-Bahr E. Susceptibility genes and modifiers for cardiac arrhythmias. Cardiovasc Res. 2005; 67: 397-413.

4. Kapplinger JD, Tester DJ, Alders M, et al. An international compendium of mutations in the SCN5A-encoded cardiac sodium channel in patients referred for Brugada syndrome genetic testing. Heart Rhythm. 2010; 7: 33-46.

5. Schmitt N, et al. Cardiac Potassium Channel Subtypes: New Roles in Repolarization and Arrhythmia. Physiological Reviews 2014; 94 : 609-653.

6. Babarova M, et al. Dual Effect of Ethanol on Inward Rectifier Potassium Current IK1 in Rat Ventricular Myocytes. J Physiol Pharmacol. 2014; 65: 497-509.

7. Kauferstein S, Kiehne N, Neumann T, et al. Cardiac gene defects can cause sudden cardiac death in young people. Dtsch Arztebl Int. 2009; 106(4): 41-47.

8. Antzelevitch C. Drug-induced spatial dispersion of repolarization. Cardiol J. 2008; 15 (2): 100-121.

9. Szczeklik A, Tendera M. Kardiologia Tom I. Kraków: Medycyna Praktyczna 2009: 447-450 (in Polish).

10. Splawski I, Timothy KW, Sharpe LM. Ca(V)1.2 calcium channel dysfunction causes a multisystem disorder including arrhythmia and autism. Cell 2004; 119: 19-31.
11. Markiewicz-Łoskot G, Moric-Janiszewska E, Mazurek U. The risk of cardiac events and genotype-based management of LQTS patients. Ann Noninvasive Electrocardiol. 2009; 14: 86-92.

12. Schwartz PJ, Moss AJ, Vincent GM, Crampton RS. Diagnostic criteria for the long QT syndrome. An update. Circulation 1993; 88: 782-784.

13. Priori SG, Wilde AA, Horie M, Cho Y, Behr ER, Berul C, et al. HRS/EHRA/APHRS Expert Consensus Statement on the Diagnosis and Management of Patients with Inherited Primary Arrhythmia Syndromes. Heart Rhythm. 2013; 10(12): 1932-1963.

14. Bos JM, Bos KM, Johnson JN, Moir C, Ackerman MJ.Left cardiac sympathetic denervation in long QT syndrome: analysis of therapeutic nonresponders. Circulation: Arrhythmia and Electrophysiology. 2013; 6: 705-711.

15. Brugada P, Brugada J. Right bundle branch block, persistent ST segment elevation and sudden cardiac death: a distinct clinical and electrocardiographic syndrome. A multicenter report. J Am Coll Cardiol. 1992; 20: 1391-1396.

16. Donohue D, Tehrani F, Jamehdor R, Lam C, Movahed MR. The prevalence of Brugada ECG in adult patients in a large university hospital in the western United States. Am Heart Hosp J. 2008; 6: 48-50.

17. Miyasaka Y, Tsuji H, Yamada K, Tokunaga S, Saito D, Imuro Y, et al. Prevalence and mortality of the Brugada-type electrocardiogram in one city in Japan. J Am Coll Cardiol. 2001; 38: 771-774.

18. Berne P1, Brugada J. Brugada Syndrome 2012. Circulation Journal 2012; 76(7): 1563-1571.

19. Nowowiejska-Wiewióra A, Hudzik B, Adamowicz-Czoch E. Zespół Brugadów - tym razem do dwóch razy sztuka. Folia Cardiologica Excerpta 2010; 5(5): 310-314 (in Polish).

20. Gaita F, Giustetto C, et al. Short QT Syndrome: a familial cause of sudden death. Circulation. 2003; 108(8): 965-970.

21. Bellocq C, van Ginneken AC, et al. Mutation in the KCNQ1 gene leading to the short QT-interval syndrome. Circulation. 2004; 109(20): 2394-2397.

22. Giustetto C, Schimpf R, et al. Long-term follow-up of patients with short QT syndrome. Journal of the American College of Cardiology. 2011; 58(6): 587-595.

23. Tester DJ, Ackerman MJ. Postmortem long QT syndrome genetic screening for sudden unexplained death in the young. J Am Coll Cardiol. 2007; 49: 240-243.

24. Vyas H, Hejlik J, Ackermann MJ. Epinephrine QT stress testing in the evaluation of congenital LQTS: diagnostic accuracy of paradoxical QT response. Circulation 2006; 113: 1385-1392. 